\title{
The Goalkeeper Influence on Ball Possession Effectiveness in Futsal
}

\author{
by \\ Pedro Vicente-Vila ${ }^{1}$ Carlos Lago-Peñas ${ }^{1}$
}

\begin{abstract}
The aim of this study was to identify which variables were the best predictors of success in futsal ball possession when controlling for space and task related indicators, situational variables and the participation of the goalkeeper as a regular field player or not (5 vs. 4 or 4 vs. 4). The sample consisted of 326 situations of ball possession corresponding to 31 matches played by a team from the Spanish Futsal League during the 2010-2011, 2011-2012 and 2012-2013 seasons. Multidimensional qualitative data obtained from 10 ordered categorical variables were used. Data were analysed using chi-square analysis and multiple logistic regression analysis. Overall, the highest ball possession effectiveness was achieved when the goalkeeper participated as a regular field player $(p<0.01)$, the duration of the ball possession was less than $10 \mathrm{~s}(p<0.01)$, the ball possession ended in the penalty area $(p<0.01)$ and the defensive pressure was low $(p<0.01)$. The information obtained on the relative effectiveness of offensive playing tactics can be used to improve team's goal-scoring and goal preventing abilities.
\end{abstract}

Key words: performance analysis, offensive performance, logistic regression, goalkeeper.

\section{Introduction}

Futsal is a variant of soccer that is played indoors and worldwide. Futsal has been growing as a relatively new sport since FIFA standardised the regulations and international competitions (i.e., the first World Championship was played in 1989, Rotterdam, the Netherlands). However, from a scientific perspective, there is a lack of research in futsal, in particular when comparing the available research with soccer.

Previous research carried out in this sport has mainly focused on physiological (Álvarez et al., 2009), injury-related (Junge and Dvorak, 2010), and psychological aspects (Geisler and Kerr, 2007). From a performance analysis perspective the available research is limited and the studies have mainly analysed a variety of tactics and match situations. On the one hand, the researchers have studied tactical approaches in futsal (Lapresa et al., 2013). These studies highlighted the importance of tactical systems and space as key indicators when finalising ball possession in futsal. On the other hand, futsal has been studied from tactical modelling and dynamic perspectives (Fonseca et al., 2013; Travassos et al., 2012).

According to the Futsal Laws of the Game (Fédération Internationale de Football Association, Law 3), any player may change places with the goalkeeper without informing the referees or waiting for a stoppage in a match. They may be substituted by a regular field player if their team elects to use this scheme in order to outnumber the defending players, that is, 5 vs. 4 . This field player becomes the designated goalkeeper on the court; and must wear some vest or bib to be identified as such. Despite this tactical possibility, up to now there is lack of research concerning the impact of playing 4 vs. 4 or 5 vs. 4 on ball possession effectiveness in futsal. In a recent study, Correa et al. (2014) examined how the goalkeeper as an outfield player affected

1 - Faculty of Education and Sport Sciences, University of Vigo, Campus Pontevedra, Spain. 
player's behaviour in futsal. They found that the goalkeeper acting as an outfield player was an effective strategy for attacking in terms of increasing shots at the goal and that each team reduced its defensive area as well as its variability in situations where the goalkeeper acted as an outfield player.

However, the available literature exploring team-tactical structures and effectiveness regarding space and task related indicators and situational variables in futsal is limited when all these factors are addressed simultaneously (Moore et al., 2014), probably due to the fact that performance analysis in futsal gathering these variables is complex and nonlinear. Therefore multivariate technique is a useful tool when describing the normative profiles of ball possession effectiveness and their association with space and task related indicators and the situational variables. Moreover, none of the aforementioned studies have examined the influence of the goalkeeper on ball possession effectiveness.

Therefore, the aim of the present study was to identify the impact of playing 4 vs. 4 or 5 vs. 4 on ball possession effectiveness in futsal and to determine the best predictors (i.e., space and task related indicators and situational variables) of success in futsal ball possession using the binomial logistic regression. It was hypothesized that ball possession effectiveness in futsal was dependent on space and task performance indicators as well as situational variables and that teams using the 5 vs. 4 pattern would obtain better results than those using 4 vs. 4 .

\section{Material and Methods}

\section{Participants}

The sample consisted of 326 situations of ball possession corresponding to 31 matches played by a team from the Spanish Futsal League during the 2010-2011, 2011-2012 and 2012-2013 seasons where the goalkeeper played as a regular field player ( 5 vs. 4,163 situations) or not (4 vs. 4 , 163 situations). In futsal, goalkeepers may participate in the normal play of their teammates. They may be substituted by a regular field player if their team elects to use this scheme in order to outnumber the defending players. This field player becomes the designated goalkeeper on the court and must wear some vest or bib to be identified as such. The matches analysed (7 playoff and 24 regular season matches) resulted in 6 wins, 7 draws, and 18 losses. The Spanish competition consists of 16 teams that vie for the national soccer championship. Teams play each other twice a season, once during rounds 1-15 and once during rounds 16-30 when the status of home and visiting team is reversed. The playoff league stage is played by the 8 teams classified best during the regular season (played in a balanced schedule of 15 teams), then the playoff includes quarter-final, semi-final and final rounds in a best-of-three-series with a home court advantage predetermined by the regular season results, the best classified team guarantees the home court advantage.

\section{Measures}

All the variables included are defined in Table 1. The dependent variable was ball possession offensive effectiveness. It was established into a dichotomous dependent variable successful ball possession (when the offensive team scored a goal) and unsuccessful ball possessions (when the offensive team did not score a goal).

The independent variables were related to space and task related variables and situational variables. The space was studied by the possession ending areas (see Figure 1) of the court (7 zones were used according to Álvarez et al., 2004).

The task related variables included: (i) participation of the goalkeeper as a regular field player or not (5 vs. 4 or 4 vs. 4); (ii) ball possession duration (from 0 to $10 \mathrm{~s}$ and more than $10 \mathrm{~s}$ ); (iii) the number of passes (from 0 to 2 passes, 3 to 5 passes and more than 5 passes); (iv) the number of players involved $(0-2$ players, 3 4 players and > 4 players); (v) existence of defensive pressure by the opponent (shooting player under pressure and shooting player under no pressure) and (vi) the number of defending players into the offensive influence zone $(0$ defenders, 1 defender and more than 1 defender). In order to control for situational variables effect, match location (playing at home or away) and match status (1 goal up, 1 goal down, 2 or more goals up, 2 or more goals down, level score) were included as independent variables.

Procedures

The matches were analysed through 
observational data notation. The observational analysis was developed by one experienced observer (graduated in Sports Sciences with ten years of experience as a coach) trained for this task during two months. In order to asses intraobserver reliability (weighted Kappa correlations coefficients) 33 randomly ball possession situations were observed again after a 4 week period (O'Donoghue and Holmes, 2015). The intra-observer reliability results were very with good kappa values (0.98) (Altman, 1991). Ethical approval from the University of Vigo was obtained.

\section{Statistical Analysis}

Firstly, a binary logistic regression model was used to assess the relationship between offensive, defensive and situational variables according to ball possession offensive effectiveness. The dependent variable used in the model was $Y \in\{0,1\}$, with 0 (1) values for unsuccessful (successful) ball possession (Willoghby, 2002). The binomial logistic regression model was expressed as follow:

$$
\begin{gathered}
\mathrm{e}^{(\mathrm{Z})} \\
\mathrm{E}(\mathrm{Y} / \mathrm{X})=-\mathrm{e}^{(\mathrm{Z})}
\end{gathered}
$$

where $\mathrm{Z}$ represents=

$$
\begin{gathered}
\beta_{0}+\beta_{1}{ }^{*} G P \beta_{2}{ }^{*} D+\beta_{3}{ }^{*} L+\beta_{4}{ }^{*} N B H+\beta_{5}{ }^{*} D e f P+ \\
\beta_{6}{ }^{*} D e f D+\beta_{7} S Z+\beta_{8}{ }^{*} S+\beta 9^{*} M L+\varepsilon i .
\end{gathered}
$$

$\beta_{0}$ is the constant of the equation and the independent variables were GP $=$ Goalkeeper
Participation, $\mathrm{D}=$ Duration, $\mathrm{L}=$ Length, NPI = Number of players involved, DefP $=$ Defensive pressure, DefD $=$ Defensive density, $\mathrm{SZ}=$ Shot zone, $S=$ Score and ML = Match Location; $\varepsilon_{i}$ was the disturbance term.

This non-linear regression model estimates the regression coefficients that represent the estimated change in the log-odds, corresponding to a unit change in the corresponding explanatory variable conditional on the other explanatory variables remaining constant (Landau and Everitt, 2004). The Odds ratios (OR) and their $95 \%$ confidence intervals (CI) were also determined.

Secondly, the chi-square test was used to identify the influence of the independent variables on ball possession effectiveness. Both statistical analyses were performed using IBM SPSS statistics for Windows version 20.0 (Armonk, NY; IBM Corp.). The level of significance was set at $p \leq 0.05$.

\section{Results}

The distribution of relative frequencies from the studied variables is shown in Table 2.

Results from the binary logistic regression analysis (Table 3) showed that there were significant relationships between ball possession effectiveness and the participation of the goalkeeper as a regular field player (5 vs. 4 ) or not $(4$ vs. 4$)(p<0.01)$, possession duration $(p<01)$, passes used $(p<.05)$, shot zone $(p<.01)$ and defensive density in the offensive influence zone $(p<.01)$.

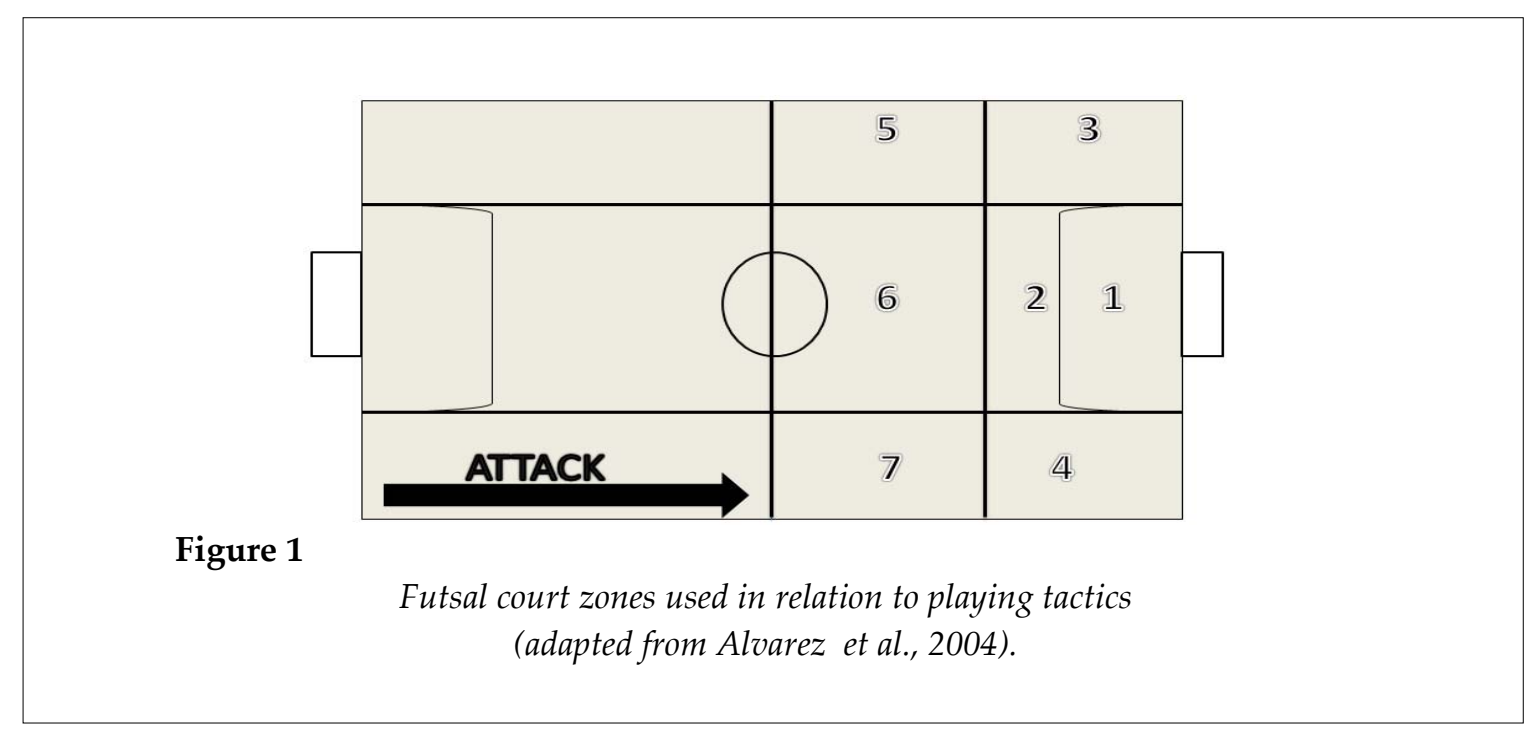

(C) Editorial Committee of Journal of Human Kinetics 
Table 1

Variables studied in elite futsal

Dependent variable

Ball possession effectiveness

The variable was established as a dichotomous variable: successful ball possession (when the offensive team scored a goal), and unsuccessful ball possession (when the offensive team did not score a goal).

\section{Independent variables}

Goalkeeper participation

The variable was established as a dichotomous variable: the goalkeeper participated as a regular field player (5 vs. 4 ) or not (4 vs. 4$)$.

Possession duration

Duration of each ball possession was gathered and divided into 2 groups: 0-10 s and more than $10 \mathrm{~s}$.

The number of passes used during the ball possession was

Passes used split into 3 groups: $0-2$ passes, $3-5$ passes and more than 5 passes.

The number of players involved during the ball possession,

Number of players involved this variable was divided into 3 groups: micro group (0 - 2 players involved), medium group ( 3 - 4 players involved) and macro group ( $>4$ players involved).

Defensive pressure

Two defensive pressure situations were analysed: a shooting player under pressure or not.

The number of defending players in the offensive influence

Defensive density zone: low defensive density ( 0 defenders), middle defensive density ( 1 defender) and high defensive density $(>1$ defenders).

Ending zone

Gathering the zone where the ball possession ended according to the court division used by Puente et al. (2004) Z1, Z2, Z3, Z4, Z5, Z6, Z7.

\section{Situational Variables}

Match Status

The variable was defined according to five possibilities: 1 goal up, 2 or more goals down, level score, 1 goal down, 2 or more goals down.

Match Location

Playing at home or away. 
Table 2

Distribution of relative frequencies from the studied variables

\begin{tabular}{|c|c|c|c|c|c|c|}
\hline Variable & Type of possession & $(n=326)$ & Goal & $\%$ & No Goal & $\%$ \\
\hline Goalkeeper & 4 vs. 4 & 163 & 19 & 11.7 & 144 & 88.3 \\
\hline participation & 5 vs. 4 & 163 & 32 & 19.6 & 131 & 80.4 \\
\hline \multirow{3}{*}{$\begin{array}{l}\text { Possession } \\
\text { duration }\end{array}$} & Fast & 211 & 41 & 19.4 & 170 & 80.6 \\
\hline & Slow & 115 & 10 & 8 & 105 & 91.3 \\
\hline & Short & 155 & 23 & 14.8 & 132 & 85.2 \\
\hline \multirow[t]{2}{*}{ Passes used } & Medium & 78 & 11 & 14.1 & 67 & 85.9 \\
\hline & Long & 93 & 17 & 18.3 & 76 & 81.7 \\
\hline \multirow{3}{*}{$\begin{array}{l}\text { Number of } \\
\text { players involved }\end{array}$} & Micro group & 120 & 14 & 11.7 & 106 & 88.3 \\
\hline & Medium group & 155 & 28 & 18.1 & 127 & 81.9 \\
\hline & Macro group & 51 & 9 & 17.6 & 42 & 82.4 \\
\hline \multirow{2}{*}{$\begin{array}{l}\text { Deffensive } \\
\text { pressure }\end{array}$} & Pressure & 116 & 12 & 10.3 & 104 & 89.7 \\
\hline & No pressure & 210 & 39 & 18.6 & 171 & 81.4 \\
\hline \multirow{7}{*}{$\begin{array}{l}\text { Deffensive } \\
\text { density }\end{array}$} & Low & 155 & 23 & 14.8 & 132 & 85.2 \\
\hline & Medium & 78 & 11 & 14.1 & 67 & 85.9 \\
\hline & High & 93 & 17 & 18.3 & 76 & 81.7 \\
\hline & Zone 1 & 89 & 42 & 47.2 & 47 & 52.8 \\
\hline & Zone 2 & 41 & 3 & 7.3 & 38 & 92.7 \\
\hline & Zone 3 & 24 & 0 & 0 & 34 & 100 \\
\hline & Zone 4 & 40 & 2 & 5 & 38 & 95 \\
\hline \multirow[t]{5}{*}{ Shot zone } & Zone 5 & 17 & 0 & 0 & 17 & 100 \\
\hline & Zone 6 & 68 & 2 & 2.9 & 66 & 97 \\
\hline & Zone 7 & 37 & 2 & 5.4 & 35 & 94 \\
\hline & 2 or more Goals & 6 & 1 & 16.7 & 5 & 83.3 \\
\hline & Down & & & & & \\
\hline \multirow[t]{4}{*}{ Score } & 1 Goal down & 21 & 3 & 14.3 & 18 & 85.7 \\
\hline & Level & 44 & 9 & 20.5 & 35 & 79.5 \\
\hline & 1 Goal Up & 83 & 17 & 20.5 & 66 & 79.5 \\
\hline & 2 or more Goals Up & 172 & 21 & 12.2 & 151 & 87.8 \\
\hline \multirow[t]{2}{*}{ Match Location } & Home & 138 & 24 & 17.4 & 114 & 82.6 \\
\hline & Away & 188 & 27 & 14.4 & 161 & 85.6 \\
\hline
\end{tabular}

Table 3

Results of success in ball possession as a function of performance indicators in a futsal team.

\begin{tabular}{ccccccccc}
\hline $\begin{array}{c}\text { Success in ball } \\
\text { possession }\end{array}$ & B & SE & Wald & df & $p$ & OR & $\begin{array}{c}\text { OR (95\% CI) } \\
\text { Lower }\end{array}$ & Upper \\
\hline $\begin{array}{c}\text { Goalkeeper } \\
\text { participation } \\
\text { Passes used }\end{array}$ & -1.34 & .48 & 7.74 & 1 & .00 & .26 & .10 & .67 \\
$\begin{array}{c}\text { Number of } \\
\text { players involved }\end{array}$ &. .95 & .44 & 4.55 & 1 & .03 & .38 & .16 & .92 \\
$\begin{array}{c}\text { Defensive pressure } \\
\text { Duration }\end{array}$ &. .55 & .44 & .27 & 1 & .59 & 1.26 & .53 & 2.99 \\
$\begin{array}{c}\text { Deffensive } \\
\text { density }\end{array}$ & .43 & 1.58 & 1 & .20 & .57 & .24 & 1.36 \\
Shot zone & .94 & .26 & 12.79 & 1 & .00 & 2.57 & 1.53 & 4.32 \\
Score & .52 & .15 & 11.38 & 1 & .00 & 1.68 & 1.24 & 2.27 \\
Match Location & .19 & .20 & .87 & 1 & .34 & 1.21 & .81 & 1.80 \\
\hline
\end{tabular}




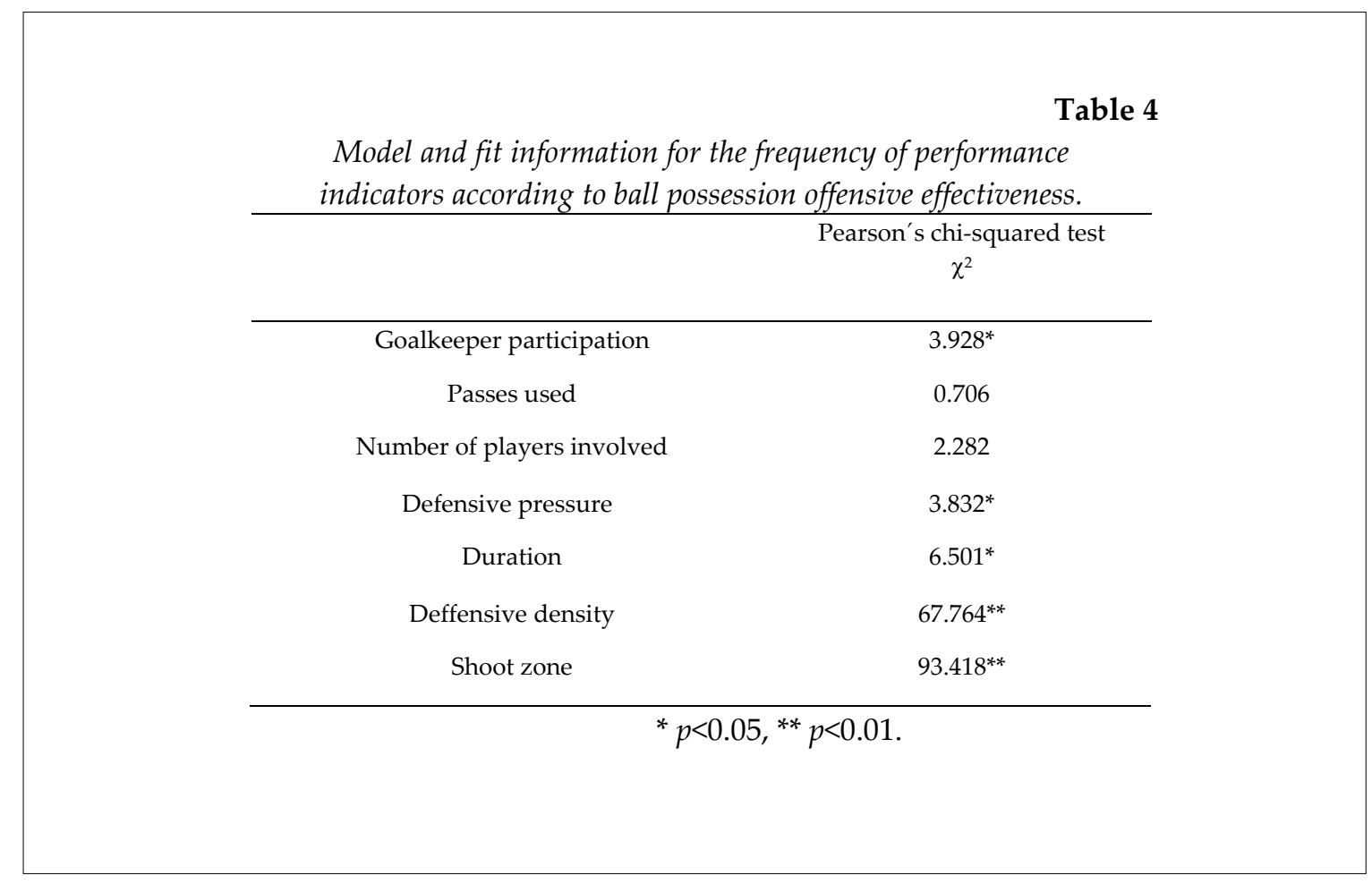

Results from the Pearson's chi-squared test indicated significant relationships between ball possession effectiveness and goalkeeper participation, defensive pressure, duration time, defensive pressure, defensive density and the shot zone (Table 4).

The highest ball possession effectiveness was achieved by the team when the goalkeeper participated as a regular field player (5 vs. 4; $p<0.05)$, the duration of the ball possession was less than $10 \mathrm{~s}(p<0.05)$, ball possession ended in the penalty area $(p<0.01)$ and the defensive pressure was low $(p<0.05)$.

\section{Discussion}

The aim of this study was to identify which variables were the best predictors of success in futsal ball possession when controlling for space and task related indicators and situational variables.

This study has strengths worthy of consideration. Firstly, up to now this is one of the first studies that have analyze the participation of the goalkeeper as a regular field player on success in futsal. Moreover, we used logistic regression, an appropriate statistical method for comparisons of categorical differences associated with binary response variables (Nevill et al., 2002).
For the main variable i.e. "goalkeeper participation", results from logistic regression analyses showed that success in ball possession was higher when the goalkeeper participated as a regular field player ( 5 vs. 4 ) than when the team played 4 vs. 4 . These findings are similar to those provided by Correa et al. (2014) who found that the goalkeeper acting as an outfield player was an effective strategy for attacking in terms of increasing the number of shots at the goal. Results from the current study showed that the probability for scoring a goal was 3.6 times higher when the team attacked with the goalkeeper as a regular field player (5 vs. 4).

The assessment of opponent interactions revealed that playing against low defensive pressure increased offensive effectiveness. The differences in the study design and variable types as well as their definitions make a direct comparison between studies that had assessed opponent interactions and situational variables difficult. Previous studies generally support the current findings. In soccer, Harris and Reilly (1988) showed that defence against attacks with a shot on target, compared with those without a shot, tended to involve higher attacker to defender ratios and greater average distances between the attacker in possession and the nearest 
defender throughout the attack. According to Grehaigne (1991), the overall attacking configuration with adequate space and time and the opponent's defence with its centre of gravity out of the position had a positive effect on the scoring of 10 of 33 goals. Again in soccer, Seabra and Dantas (2006) reported a higher proportion of successful shooting attempts for ball receptions and shots originating from zones of low defensive confrontation than of high defensive confrontation. Additionaly, Olsen et al. (1997) showed more scoring opportunities and goals from breakdown attacks (counterattacks) started when the opposition defence was imbalanced rather than balanced. As proposed by Tenga et al. (2010), the tactics of balanced defence (tight pressure, present backup and present cover) are more effective in preventing score-box possession than the opposite tactics of imbalanced defence (loose pressure, absent backup and absent cover).

For the duration variable, results showed that short possession was more effective than long possession. To our knowledge, no studies had examined this variable before. The current finding is in accordance with some previous studies in soccer (Bate, 1988; Reep and Benjamin, 1968), but different from those of Tenga et al. (2010) and Lago-Ballesteros et al. (2012). Data from the study of Reep and Benjamin (1968) and Hughes and
Franks (2005) demonstrated that more shots were indeed produced from shorter passing sequences.

Significant differences were found when analysing success in ball possession and the area in which the possession ended. The highest ball possession effectiveness was achieved when the teams ended ball possession in the penalty area (zone 1) and they were the ones which had the highest rate of occurrence $(47.2 \%)$. These results are similar to those provided by Lapresa et al. (2013) and Álvarez et al. (2004) who found that approximately $80 \%$ of the shots that achieved a goal came from within $10 \mathrm{~m}$ of the goal.

The unexpected non-significant independent influence of the match location and match status on success in ball possession probability could be explained by the fact that situational variables would have unique effects on individual players, teams and playing styles (Barnett and Hilditch, 1993; Bloomfield et al., 2005; Clarke and Norman, 1995; Lago, 2009).

In terms of the limitations of the present study, the attacking performance of a single elite soccer team was analysed and consequently the results obtained could be a reflection of the playing standard or style of this particular team, so care should be taken when extrapolating these results to other teams.

\section{References}

Altman D. Practical statistics for medical research. CRC Press; 1990

Álvarez J, D'Ottavio S, Vera J, Castagna C. Aerobic fitness in futsal players of different competitive level. J Strength Cond Res, 2009; 23(7): 2163-2166

Álvarez J, Manero J, Manolelles P, Puente J. Analysis of the offensive actions resulting in goal of professional league of Spanish futsal. Revista de entrenamiento deportivo, 2004; 23(4): 27-32

Barnett $\mathrm{V}$, Hilditch $\mathrm{S}$. The effect of an artificial pitch surface on home team performance in football (soccer). $J$ Roy Statistical Soc B, 1993; 156: 39-50

Bate R. Football chance: Tactics and strategy. In: T. Reylly, A. Lees, K. Davids \& W.J. Murphy (Eds.) Science and football. London: E \& FN Spon, 293-301; 1988

Bloomfield L, Polman R, O'Donoghue PG. Effects of core - line on team strategies in FA Premier Soccer League. J Sport Sci, 2005; 23: 192-193

Clarke S, Norman J. Home ground advantage of individual clubs in English soccer. J Roy Stat Soc D, 1995; 44(4): 509-521

Corrêa UC, Davids K, Silva SL, Denardi RA, Tani G. The Influence of a Goalkeeper as an Outfield Player on Defensive Subsystems in Futsal. Advances in Physical Education, 2014; 4: 84-92

Fonseca S, Milho J, Travassos B, Araújo D, Lopes A. Measuring spatial interaction behavior in team sports 
using superimposed Voronoi diagrams. Int J Perf Anal Sport, 2013; 13(1): 179-189

Geisler G, Kerr JH. Competition stress and affective experiences of Canadian and Japanese futsal players. Int J Sport Psychol, 2007; 38(2): 187-206

Greghaine JF. A new method of goal analysis. Science and Football, 1991; 5(10): 12-16

Harris S, Reilly T. Space, teamwork and attacking success in soccer. In: T. A. Lees, K. Davis \& W. Murphy (Eds.), Science and Football. London - New York: E. \& F.N. Sport, 322-328; 1988

Hughes M, Franks I. Analysis of passes sequences, shots and goals in soccer. J Sport Sci, 2005; 23(5): 509-514

Junge A, Dvorak J. Injury risk of playing football in Futsal World Cups. Brit J Sport Med, 2010; 44: 1089-1092

Lago C. The influence of match location, quality of opposition and match status on possession strategies in professional association football. J Sport Sci, 2009; 27(13): 1463-1469

Lago - Ballesteros J, Lago - Peñas C, Rey E, Casáis L, Domínguez E. The offensive success in high level football. The influence of tactics models used and situational variables. European J Hum Movement, 2012; 28: 145-170

Landau S, Everitt B. A handbook of statistical analyses using SPSS (Vol. 1). Boca Raton, FL: Chapman \& Hall/CRC; 2004

Lapresa D, Alvárez L, Arana J, Garzón B, Valvanera C. Observational analysis of the offensive sequences that ended in a shot by the winning team of the 2010 UEFA Futsal Championship. J Sport Sci, 2013; 31(15): 1731-1739

Moore R, Bullough S, Goldsmith S, Edmondson L. A Systematic Review of Futsal Literature. Am J Sport Med, 2014; 2(3): 108-116

Nevill A, Atkinson G, Hughes M, Cooper S. Stadistical methods for analysing discrete and categorical data recorded in performance analysis. J Sport Sci, 2012; 20(10): 829-844

O'Donoghue P, Holmes L. Data analysis in sport. Routledge; 2014

Olsen E, Larsen O, Reilly T, Bangsbo J. Use of analysis by coaches. In: T. Reilly, J. Bangsbo and M. Hughes. Science and football III. London-New York: E. y F.N. Spon; 209-220; 1997

Reep C, Benjamin B. Skill and chance in association football. J Roy Stat A, 1968; 131: 581-585

Seabra F, Dantas L. Space definition for match analysis in soccer. Int J Perf Anal Sport, 2006; 6(2): 97-113

Tenga A, Holmes I, Ronglan L, Bahr R. Effect of playing tactics on achieving score box possessions in a random series of team possessions from Norwegian professional soccer matches. J Sport Sci, 2010; 28(3): 245-255

Travassos B, Araújo D, Davids K, Vilar L, Esteves P, Vanda C. Informational constraints shape emergent functional behaviours during performance of interceptive actions in team sports. Psych J Sport Ex, 2012; 13(2): 216-223

Willoughby KA. Winning games in Canadian football: A logistic regression analysis. Coll Math J, 2002; 33(3): $215-220$

\section{Corresponding author:}

\section{Pedro Vicente Vila}

Faculty of Education and Sport Sciences, University of Vigo, Campus Pontevedra, Spain.

Campus da Xunqueira s/n - 36005 - Pontevedra - Galicia - España.

Phone :652631265 - Tlf: 986106698

Fax: 986304047

E-mail: pachipedrito@edu.xunta.es 